\title{
FERTILIDADE DO SOLO EM INTEGRAÇÃO LAVOURA-PECUÁRIA NA REGIÃO DE GUARAPUAVA/PR
}

\author{
Fertility of the Soil in Crop-Cattle Raising \\ Integration in Guarapuava
}

\author{
Denise Bruginski de Carvalho ${ }^{1}$ \\ Márcia Bello ${ }^{1}$ \\ Amir Pissaia ${ }^{2}$ \\ Aníbal de Mora es ${ }^{2}$ \\ Adelino Pelissari ${ }^{2}$ \\ Renato Marques ${ }^{3}$ \\ Amadeu Bona Filho ${ }^{4}$
}

\section{Resumo}

O trabalho foi realizado com o objetivo de verificar o nível de fertilidade em diferentes profundidades dos solos e em duas épocas distintas em propriedades rurais da região de Guarapuava/PR que utilizam a integração lavoura-pecuária. Foram amostradas três profundidades $(0 \mathrm{a} 5 \mathrm{~cm}, 5$ a $10 \mathrm{~cm}$ e $10 \mathrm{a} 20 \mathrm{~cm}$ ), coletando-se 20 amostras simples para formação de uma amostra composta por área para análise de $\mathrm{pH}, \mathrm{Al}^{+3}$ $\left(\mathrm{cmol}_{\mathrm{c}^{\prime}} \cdot \mathrm{dm}^{-3}\right), \mathrm{Ca}^{+2}+\mathrm{Mg}^{+2}\left(\mathrm{cmol} \cdot \mathrm{dm}^{-3}\right), \mathrm{K}^{+}\left(\mathrm{cmol}_{c^{\prime}} \cdot \mathrm{dm}^{-3}\right), \mathrm{P}\left(\mathrm{mg} \cdot \mathrm{dm}^{-3}\right), \mathrm{C}\left(\mathrm{g} \cdot \mathrm{dm}^{-3}\right)$ e saturação por bases $(\mathrm{V} \%)$. O nível de fertilidade dos solos estudados, representado principalmente pelos resultados da saturação por bases, melhorou após um ano de monitoramento. Durante o período ocorreu elevação de $\mathrm{pH}, \mathrm{K}^{+2}$, C e V \%, enquanto o teor de $\mathrm{Al}^{+3}, \mathrm{Ca}^{+2}+\mathrm{Mg}^{+2}$ e $\mathrm{P}$ permaneceram constantes nas áreas estudadas. O nível de fertilidade dos solos aumentou após um ano de manejo em sistema de integração lavoura-pecuária, quando foram adotados procedimentos técnicos adequados.

Palavras-chave: Características Químicas do Solo; Propriedades Agropecuárias; Sistemas de Produção.

\section{Abstract}

This work was carried out with objective to verify the fertility level in different depths of the soils, in two different dates, in rural properties of crop-cattle raising integration in Guarapuava/PR. Three depths $(0-5 \mathrm{~cm}$, $5-10 \mathrm{~cm}$ and $10-20 \mathrm{~cm}$ ) were sampled, collecting one sample of 20 plots in the area, for $\mathrm{pH}, \mathrm{Al}^{+3}\left(\mathrm{cmol}_{\mathrm{c}} \cdot \mathrm{dm}^{-}\right.$ $\left.{ }^{3}\right), \mathrm{Ca}^{+2}+\mathrm{Mg}^{+2}\left(\mathrm{cmol}_{\mathrm{c}} \cdot \mathrm{dm}^{-3}\right), \mathrm{K}^{+}\left(\mathrm{cmol}_{\mathrm{c}} \cdot \mathrm{dm}^{-3}\right), \mathrm{P}\left(\mathrm{mg} \cdot \mathrm{dm}^{-3}\right), \mathrm{C}\left(\mathrm{g} \cdot \mathrm{dm}^{-3}\right)$ analysis and saturation bases $(\mathrm{V} \%)$. The level of fertility of the studied soils, represented mainly by the results of the saturation bases, was considered better, after one year of observation. During the experiment period it was observed an increase of the $\mathrm{pH}$, $\mathrm{K}^{+2}$, C e V \%, while the content of $\mathrm{Al}^{+3}, \mathrm{Ca}^{+2}+\mathrm{Mg}^{+2}$ e P remained constant in the studied areas. The level of fertility of soils increased after one year in crop-cattle raising integration system, because of the adoption of adequate technical procedures.

Keywords: Agricultural Properties; Production Systems; Soils Chemical Properties.

\footnotetext{
Engenheira Agrônoma, M.Sc., aluna de doutorado. Pós-graduação em Agronomia - Produção Vegetal, UFPR.

Engenheiro Agrônomo, Dr. Professor do Departamento de Fitotecnia, UFPR.

Engenheiro Florestal, Dr. Professor do Departamento de Solos, UFPR.

4 Médico Veterinário, Dr. Professor do Departamento de Nutrição Animal, UFPR.
} 


\section{Introdução}

Por meio da integração lavoura-pecuária busca-se a diversificação dos sistemas de produção e a superação de problemas advindos dos cultivos anuais sucessivos. Um dos benefícios obtidos com esta integração é que o aumento da fertilidade do solo pela adubação de lavouras em sistemas de plantio direto cria condições no solo para o desenvolvimento de pastagem de elevado potencial de produção e ótima qualidade (MORAES et al., 2000).

O aproveitamento de um mesmo nutriente para produção animal e vegetal reduz as perdas e a contaminação ambiental. Além disso, nestes sistemas integrados o retorno de nutrientes, via excrementos, dá-se diretamente sobre a área cultivada, eliminando, dessa forma, a necessidade de transporte deste material (ASSMANN et al., 2002).

Devido à necessidade da adoção de uma abordagem mais analítica e explicativa da dinâmica de produção de pastagens, visando a garantir a otimização da produção animal e a sustentabilidade dos sistemas (NABINGER et al., 1999), a Universidade Federal do Paraná vem desenvolvendo projetos na região de Guarapuava em parceria com diversas instituições, promovendo discussões interdisciplinares sobre a adoção e o manejo do sistema de integração lavoura-pecuária.

Diferentes aspectos envolvidos no sistema foram objetos de estudo, como, por exemplo, o ganho de peso animal, a inclusão de leguminosas nas misturas forrageiras, a nutrição mineral e produção de massa seca de forrageiras, a produtividade de lavouras associada à fertilização do sistema e as características físicas do solo (MORAES et al., 2002).

A integração lavoura-pecuária apresentase como uma estratégia muito promissora para desenvolver sistemas de produção menos intensivos no uso de insumos e mais sustentáveis no tempo (ASSMANN et al., 2002).

Partindo-se do pressuposto de que a ciclagem dos nutrientes é otimizada em sistema de integração lavoura-pecuária, quando este é associado com o sistema de plantio direto e a rotação de culturas, então a fertilidade do solo pode ser melhorada com o tempo.

O objetivo deste trabalho é verificar o nível de fertilidade em diferentes profundidades dos solos submetidos ao sistema de integração lavoura-pecuária, em duas épocas distintas, em diferentes propriedades rurais da região de Guarapuava/PR.

\section{Materiais e Métodos}

Os dados foram coletados na Região de Guarapuava/PR, em áreas selecionadas dentro de seis fazendas que utilizam o sistema de integração lavoura-pecuária, denominadas com letras "A", "B", "C", "D", "E" e "F". A área dos talhões monitorados, a granulometria do solo e as características do pastejo das seis propriedades estudadas estão apresentadas na Tabela 1 .

\section{TABELA 1 - Área dos talhões monitorados, granulometria do solo e características do pastejo no sistema de integração lavoura-pecuária em seis propriedades rurais da região de Guarapuava/PR. 2001.}

Table 1 - Area of the monitored stands, soil grain size and grazing characteristics in crop-cattle raising integration system in six properties of the Guarapuava/PR region. 2001.

\begin{tabular}{|c|c|c|c|c|c|}
\hline \multirow[t]{2}{*}{ Propriedade } & \multirow{2}{*}{$\begin{array}{l}\text { Area } \\
\text { (ha) }\end{array}$} & \multicolumn{3}{|c|}{ Granulometria do so lo } & \multirow[t]{2}{*}{ Sistema de paste jo durante o pe ríodo de inve mo } \\
\hline & & $\begin{array}{c}\text { Areia } \\
(\%)\end{array}$ & $\begin{array}{l}\text { Silte } \\
(\%)\end{array}$ & $\begin{array}{c}\text { Argila } \\
(\%)\end{array}$ & \\
\hline $\mathrm{A}$ & 36 & 0,6 & 39,4 & 60 & Rotacionado com carga fixa ( 3 cabeças ha ch $^{-1}$ ) \\
\hline $\mathrm{B}$ & 9 & 2,2 & 39,8 & 58 & Contínuo com carga fixa ( 5 cabeças $\mathrm{ha}^{-1}$ ) \\
\hline $\mathrm{C}$ & 11 & 4,6 & 53,4 & 42 & Contínuo com carga fixa $\left(4,5\right.$ cabeças ha $\left.^{-1}\right)$ \\
\hline $\mathrm{D}$ & 5 & 2,2 & 39,6 & 58 & Contínuo corn carga variável \\
\hline $\mathrm{E}$ & 17 & 2,4 & 39,6 & 58 & Rotacionado com carga fixa ( 3 cabeças ha $^{-1}$ ) \\
\hline $\mathrm{F}$ & 4 & 2,6 & 35,4 & 62 & Rotacionado com carga variável \\
\hline
\end{tabular}


Com exceção do solo da propriedade

F que é classificado como muito argiloso, os demais se classificam na categoria de solo argiloso (TOMÉ JÚNIOR, 1997).
A rotação de culturas nas diferentes áreas monitoradas e as quantidades de calcário, $\mathrm{N}_{2} \mathrm{P}_{2} \mathrm{O}_{5}$ e $\mathrm{K}_{2} \mathrm{O}$ adicionadas ao solo durante o período de estudo são apresentadas na Tabela 2.

\section{TABELA 2 - Cultivo de verão (2000/01) e de inverno (2001), fertilização com $\mathrm{N}, \mathrm{P}_{2} \mathrm{O}_{5}$ e $\mathrm{K}_{2} \mathrm{O}$ e, quantidade de calcário aplicado no sistema de integração lavoura-pecuária em seis pro- priedades rurais da região de Guarapuava/PR. 2001.}

Table 2 - Summer (2000/01) and winter (2001) crop, fertilization with $\mathrm{N}, \mathrm{P}_{2} \mathrm{O}_{5}$ and $\mathrm{K}_{2} \mathrm{O}$ and, amount of calcareous rock applied in crop-cattle raising integration system in six properties of the Guarapuava/PR region. 2001.

\begin{tabular}{ccccccc}
\hline Proprie dade & & Cultivo & \multicolumn{2}{c}{ Fertilizção $\left(\mathrm{kg}^{\left.-\mathrm{ha}^{-1}\right)}\right.$} & \multicolumn{2}{c}{ Calcánio } \\
& Verão & Inverno & $\mathrm{N}$ & $\mathrm{P}_{2} \mathrm{O}$, & $\mathrm{K}_{2} \mathrm{O}$ & $\left(\mathrm{kg} \cdot \mathrm{ha}^{-1}\right)$ \\
\hline $\mathrm{A}$ & Milho & Aveia & 175 & 191 & 146 & 2000 \\
$\mathrm{~B}$ & Sorgo + rilhe to & Aveia + azevérm & 253 & 68 & 45 & - \\
$\mathrm{C}$ & Milho & Aveia + azevém + trevo & 258 & 130 & 100 & 3000 \\
$\mathrm{D}$ & Soja & Aveia + azevém + trevo & 100 & 57 & 38 & 2000 \\
$\mathrm{E}$ & Milho & Aveia + azevém + trevo & 275 & 120 & 80 & - \\
$\mathrm{F}$ & Sorgo & Aveia + azevém + trevo & 200 & 112 & 160 & 2500 \\
\hline
\end{tabular}

A fertilização apresentada na Tabela 2 é o total dos elementos adicionados ao sistema na instalação de culturas e em cobertura durante o período de estudo (dezembro de 2000 a dezembro de 2001). Nas propriedades B e F a correção de acidez pela adição de calcário havia sido realizada no ano de 1999.

A coleta de solo para análise foi realizada em duas épocas distintas, sendo a primeira em dezembro de 2000 e a segunda em dezembro de 2001, por meio da utilização de trado calador. Foram amostradas três profundidades $(0 \mathrm{a} 5 \mathrm{~cm}, 5 \mathrm{a}$ $10 \mathrm{~cm}$ e 10 a $20 \mathrm{~cm}$ ), coletando-se 20 amostras simples para formação de uma amostra composta por área. As análises de $\mathrm{pH}, \mathrm{Al}^{+3}\left(\mathrm{cmol}_{\mathrm{c}^{\prime}} \cdot \mathrm{dm}^{-3}\right), \mathrm{Ca}^{+2}$ $+\mathrm{Mg}^{+2}\left(\mathrm{cmol}_{\mathrm{c}} \cdot \mathrm{dm}^{-3}\right), \mathrm{K}^{+}\left(\mathrm{cmol}_{\mathrm{c}} \cdot \mathrm{dm}^{-3}\right), \mathrm{P}\left(\mathrm{mg}^{-3} \mathrm{dm}^{-3}\right)$, $\mathrm{C}\left(\mathrm{g} . \mathrm{dm}^{-3}\right)$ e saturação por bases (V \%) foram realizadas no Laboratório de Fertilidade de Solos da UFPR.
Para análise estatística dos dados, considerou-se o delineamento de parcela subdividida no tempo com o fator principal (profundidade de amostragem) disposto em blocos casualizados com seis repetições (propriedades) e as subparcelas representadas pelos dois anos de amostragem. Utilizou-se o programa estatístico SANEST e as médias dos tratamentos com diferença significativa pelo teste $\mathrm{F}$ foram submetidas ao teste de Tukey ao nível de 5\% de significância.

\section{Resultados}

O resultado da análise estatística dos dados demonstrou que na camada superficial do solo (0 a $5 \mathrm{~cm}$ ), de um ano para outro ocorreu elevação do pH, dos teores de potássio, carbono e da saturação por bases. Os teores de alumínio, cálcio mais magnésio e fósforo permaneceram constantes (Tabelas 3 e 4). 
TABELA 3 - Resultado da análise de $\mathrm{pH}, \mathrm{Al}^{+3}, \mathrm{Ca}^{+2}+\mathrm{Mg}^{+2}$ e $\mathrm{K}^{+}$em duas épocas e três profundidades de solos no sistema de integração lavoura-pecuária em seis propriedades rurais da região de Guarapuava/PR. 2001.

Table 3 - Result of the analysis of $\mathrm{pH}, \mathrm{Al}^{+3}, \mathrm{Ca}^{+2}+\mathrm{Mg}^{+2}$ and $\mathrm{K}^{+}$at two times and three depths of soil in crop-cattle raising integration system in six properties of the Guarapuava/PR region. 2001.

\begin{tabular}{|c|c|c|c|c|c|c|c|c|}
\hline \multirow{2}{*}{$\begin{array}{l}\text { Profundidade } \\
\text { (crm) }\end{array}$} & \multicolumn{2}{|c|}{$\begin{array}{c}\mathrm{pH} \\
\left(\mathrm{CaCl}_{2}\right)\end{array}$} & \multicolumn{2}{|c|}{$\begin{array}{c}\mathrm{Al}^{+5} \\
\left(\mathrm{cmol}_{6} \cdot \mathrm{dm}^{-3}\right)\end{array}$} & \multicolumn{2}{|c|}{$\begin{array}{l}\mathrm{Ca}^{+1}+\mathrm{Mg}^{+1} \\
\left(\mathrm{cmol}_{6} \cdot \mathrm{dr}^{-3}\right)\end{array}$} & \multicolumn{2}{|c|}{$\begin{array}{c}\mathrm{K}^{+} \\
\left(\mathrm{cmol}_{6} \cdot \mathrm{dm}^{-3}\right)\end{array}$} \\
\hline & 2000 & 2001 & 2000 & 2001 & 2000 & 2001 & 2000 & 2001 \\
\hline $00-05$ & $5,1 \mathrm{Ba}$ & $5,4 \mathrm{Aa}$ & $0,03 \mathrm{Ab}$ & $0,00 \mathrm{Ab}$ & $9,2 \mathrm{Aa}$ & $11,6 \mathrm{Aa}$ & $0,24 \mathrm{Ba}$ & $0,30 \mathrm{Aa}$ \\
\hline $05-10$ & $5,1 \mathrm{Ba}$ & $5,4 \mathrm{Aa}$ & $0,05 \mathrm{Ab}$ & $0,00 \mathrm{Ab}$ & $8,2 \mathrm{Aa}$ & $9,4 \mathrm{Aab}$ & $0,21 \mathrm{Aa}$ & $0,20 \mathrm{Ab}$ \\
\hline $10-20$ & $4,8 \mathrm{~B} \mathrm{~b}$ & $5,1 \mathrm{Ab}$ & $0,36 \mathrm{~A} \mathrm{a}$ & $0,18 \mathrm{~B} \mathrm{a}$ & $5,1 \mathrm{Ab}$ & $7,1 \mathrm{Ab}$ & $0,14 \mathrm{Ab}$ & $0,16 \mathrm{Ab}$ \\
\hline Média & $5,0 \mathrm{~B}$ & $5,3 \mathrm{~A}$ & $0,15 \mathrm{~A}$ & $0,06 \mathrm{~A}$ & $7,5 \mathrm{~A}$ & $9,3 \mathrm{~A}$ & $0,19 \mathrm{~A}$ & $0,22 \mathrm{~A}$ \\
\hline $\mathrm{CV}_{(\mathrm{p} f)}(\%)$ & \multicolumn{2}{|c|}{2,62} & \multicolumn{2}{|c|}{5,68} & \multicolumn{2}{|r|}{15,30} & \multicolumn{2}{|c|}{17,23} \\
\hline $\mathrm{CV}_{\text {(nam })}(\%)$ & \multicolumn{2}{|c|}{3,46} & \multicolumn{2}{|c|}{4,62} & \multicolumn{2}{|r|}{23,98} & \multicolumn{2}{|c|}{21,14} \\
\hline
\end{tabular}

Médias seguidas por letras distintas minúsculas nas colunas e maiúsculas nas linhas diferem entre si ao nível de 5\% de significância para teste de Tukey.

A deposição de esterco animal por ocasião do pastejo parece favorecer a redução da acidez do solo, como foi relatado anteriormente (ASSMANN et al., 2002; WONG et al., 1998). As quantidades de calcário aplicadas no sistema (Tabela 2) associadas às diferentes ro- tações de culturas e sistemas de pastejo adotados nas propriedades (Tabela 1), embora apresentem diferenças, de maneira geral elevaram o pH dos solos. No entanto, são poucos os trabalhos que exploram o tema calagem num ecossistema envolvendo solo, planta e animal, podendo-se apenas considerar a importância da pressão de pastejo na dinâmica do calcário aplicado superficialmente sobre o solo (ASSMANN et al., 2002).

\section{TABELA 4 - Resultado da análise de P, C e saturação por bases (V\%) em duas épocas e três profundidades de solos no sistema de integração lavoura-pecuária em seis propriedades rurais da região de Guarapuava/PR. 2001.}

Table 4 - Result of the analysis of P, C and saturation for bases (V\%) at two times and three depths of soil in crop-cattle raising integration system in six properties of the Guarapuava/PR region. 2001.

\begin{tabular}{ccccccc}
\hline \multirow{2}{*}{$\begin{array}{c}\text { Profundidade } \\
\text { (cm) }\end{array}$} & \multicolumn{2}{c}{$\begin{array}{c}\mathrm{P} \\
\left(\mathrm{mg}^{-3} \mathrm{dm}^{-3}\right)\end{array}$} & \multicolumn{2}{c}{$\begin{array}{c}\mathrm{C} \\
\left(\mathrm{g} \cdot \mathrm{dm}^{-3}\right)\end{array}$} & \multicolumn{2}{c}{$\begin{array}{c}\mathrm{V} \\
(\%)\end{array}$} \\
\cline { 2 - 7 } & 2000 & 2000 & 2000 & 2001 & 2000 & 2001 \\
\hline $00-05$ & $5,98 \mathrm{Aa}$ & $9,87 \mathrm{Aa}$ & $34,22 \mathrm{Ba}$ & $39,75 \mathrm{Aa}$ & $55,60 \mathrm{Ba}$ & $69,60 \mathrm{Aa}$ \\
$05-10$ & $5,87 \mathrm{Aa}$ & $6,73 \mathrm{Aab}$ & $33,20 \mathrm{Aa}$ & $35,52 \mathrm{Aab}$ & $52,51 \mathrm{Aa}$ & $59,14 \mathrm{Aab}$ \\
$10-20$ & $2,22 \mathrm{Aa}$ & $4,55 \mathrm{Ab}$ & $29,40 \mathrm{Aa}$ & $31,50 \mathrm{Ab}$ & $36,66 \mathrm{Bb}$ & $49,15 \mathrm{Ab}$ \\
Média & $4,69 \mathrm{~A}$ & $7,05 \mathrm{~A}$ & $32,27 \mathrm{~A}$ & $35,59 \mathrm{~A}$ & $48,26 \mathrm{~B}$ & $59,30 \mathrm{~A}$ \\
\hline $\mathrm{CV}_{\text {ipofi }}(\%)$ & & 24,19 & & 6,34 & & 9,44 \\
\hline $\mathrm{CV}_{\text {inwo }}(\%)$ & & 54,41 & & 12,30 & & 14,12 \\
\hline
\end{tabular}

Médias seguidas por letras distintas minúsculas nas colunas e maiúsculas nas linhas diferem entre si ao nível de 5\% de significância para teste de Tukey.

A elevação do teor de carbono indica um acréscimo do teor de matéria orgânica do solo obtido multiplicando-se o teor de carbono por 1,72. O sistema de plantio direto adotado juntamente com a integração lavoura-pecuária pode ser um fator de elevação do teor de matéria orgânica dos solos, como já foi mencionado por outros autores (ASSMANN et al., 2002; BRUGINSKI, 1999; SANTOS et al., 2001). 
O elevado teor de matéria orgânica encontrada (maior que $50 \mathrm{~g} . \mathrm{dm}^{-3}$ ) poderia exercer uma maior resistência à variação do $\mathrm{pH}$, fato que não ocorreu nos solos estudados. O valor de $\mathrm{pH}$ foi elevado de 5,1 a 5,4, porém permanecendo na classe de acidez média (TOMÉ JúNIOR, 1997).

A quantidade de nutrientes retornados via fezes e urina dos animais em pastejo varia amplamente em função da qualidade e quantidade da forragem por eles consumida e também da necessidade do animal. Uma parte significativa dos nutrientes contidos numa forragem retorna à pastagem nas excreções dos animais em pastejo (LOTERO et al., 1966).

Na segunda época analisada, não foi detectada presença de alumínio tóxico na camada de 0 a $10 \mathrm{~cm}$, o que é bastante favorável aos cultivos vegetais de maneira geral. A neutralização da acidez por meio da aplicação de corretivos reduz o teor de alumínio tóxico. Assim, pode-se dizer que a prática da calagem, apesar de ser feita superficialmente no sistema de plantio direto, mantém teores de $\mathrm{Al}^{+3}$ próximos de zero, principalmente nas camadas mais superficiais.

Também os teores de cálcio e magnésio são considerados altos e certamente foram influenciados pela prática da calagem. Os teores de Ca e Mg são utilizados para o cálculo da soma de bases, que é utilizada para o cálculo da capacidade de troca catiônica que, por sua vez, entra no cálculo da saturação por bases (V\%). Embora os níveis de $\mathrm{Ca}^{+2}+\mathrm{Mg}^{+2}$ tenham permanecido constantes de um ano para outro, o aumento do V\% foi influenciado pelo significativo acréscimo do teor de potássio nos solos.

Os solos estudados entram na classe de solo eutrófico nas camadas de 0 a $10 \mathrm{~cm}$ (TOMÉ JÚNIOR, 1997). Já que a saturação por bases é um excelente indicativo das condições gerais da fertilidade do solo, sob este aspecto, os solos submetidos à integração lavoura-pecuária apresentam tendência à sustentabilidade no tangente à fertilidade. Também, a maior quantidade de resíduos sobre a superfície do solo e o aumento da atividade microbiana em sistema de plantio direto beneficia as propriedades químicas do solo (VALPASSOS et al., 2001).

As quantidades de $\mathrm{K}_{2} \mathrm{O}$ aplicadas nas diferentes propriedades variaram de 38 a $160 \mathrm{~kg}$ de $\mathrm{K}_{2} \mathrm{O} \mathrm{ha}^{-1}$ (Tabela 2), mas os teores de potássio encontrados são considerados médios (TOMÉ JÚNIOR, 1997).

O teor de fósforo permaneceu constante no período analisado, apesar da elevação de valores médios deste nutriente. O elevado coeficiente de variação desta variável indica a grande heterogeneidade dos solos das fazendas com relação ao teor de fósforo. Na segunda data de coleta de amostra, observou-se maior concentração do nutriente nas camadas mais superficiais resultantes da adubação fosfatada na linha de semeadura, o que já foi constatado em outros estudos (RHEINHEIMER; ANGHINONI, 2001; VALPASSOS et al., 2001). A presença dos animais em pastejo em sistema de integração lavoura-pecuária pode manter e melhorar os níveis de fósforo dos solos (ASSMANN et al., 2002).

As variáveis $\mathrm{pH}, \mathrm{P}, \mathrm{Ca}^{+2}+\mathrm{Mg}^{+2}, \mathrm{~K}^{+}, \mathrm{C}$ e V $\%$ apresentaram decréscimo dos valores observados proporcionalmente ao aumento da profundidade de solo amostrada. Estes resultados estão dentro do esperado com relação à variação de nutrientes no perfil de um solo agrícola.

Para a visualização das diferenças ocorridas entre as propriedades estudadas, os resultados foram apresentados nas Tabelas 5 e 6. 


\section{TABELA 5 - Resultado de análise de $\mathrm{pH}, \mathrm{Al}^{+3}, \mathrm{Ca}^{+2}+\mathrm{Mg}^{+2}$ e $\mathrm{K}^{+}$em duas épocas e três profun- didades de solos no sistema de integração lavoura-pecuária em seis propriedades rurais da região de Guarapuava/PR. 2001.}

Table 5 - Result of analysis of $\mathrm{pH}, \mathrm{Al}+3, \mathrm{Ca}+2+\mathrm{Mg}+2$ and $\mathrm{K}+$ at two times and three depths of soil in crop-cattle raising integration system in six properties of the Guarapuava/PR region. 2001.

\begin{tabular}{|c|c|c|c|c|c|}
\hline $\begin{array}{c}\text { Epoca } \\
\text { (mêsiano) }\end{array}$ & $\begin{array}{l}\text { Profundidade } \\
\text { (cm) }\end{array}$ & $\begin{array}{c}\mathrm{pH} \\
\left(\mathrm{CaCl}_{2}\right)\end{array}$ & $\begin{array}{c}\mathrm{Al}^{+5} \\
\left(\mathrm{cmol}_{8} \cdot \mathrm{dm}^{-3}\right)\end{array}$ & $\begin{array}{l}\mathrm{Ca}^{+1}+\mathrm{Mg}^{+1} \\
\left(\mathrm{cmol}_{6} \cdot \mathrm{dm}^{-3}\right)\end{array}$ & $\begin{array}{c}\mathrm{K}^{+} \\
\left(\mathrm{cmol}_{6} \cdot \mathrm{dm}^{-3}\right)\end{array}$ \\
\hline \multicolumn{6}{|c|}{ Propriedade A } \\
\hline $12 / 00$ & $00-05$ & 5,00 & 0,00 & 7,50 & 0,28 \\
\hline $12 / 01$ & $00-05$ & 5,20 & 0,00 & 9,40 & 0,44 \\
\hline $12 / 00$ & $05-10$ & 4,80 & 0,30 & 5,30 & 0,27 \\
\hline $12 / 01$ & $05-10$ & 5,30 & 0,00 & 7,80 & 0,34 \\
\hline $12 / 00$ & $10-20$ & 4,70 & 0,60 & 3,60 & 0,16 \\
\hline $12 / 01$ & $10-20$ & 4,80 & 0,40 & 4,50 & 0,25 \\
\hline \multicolumn{6}{|c|}{ Propriedade B } \\
\hline $12 / 00$ & $00-05$ & 4,90 & 0,20 & 12,70 & 0,19 \\
\hline $12 / 01$ & $00-05$ & 5,20 & 0,00 & 11,40 & 0,17 \\
\hline $12 / 00$ & $05-10$ & 5,10 & 0,00 & 11,10 & 0,13 \\
\hline $12 / 01$ & $05-10$ & 5,20 & 0,00 & 11,60 & 0,13 \\
\hline $12 / 00$ & $10-20$ & 4,50 & 0,80 & 6,00 & 0,12 \\
\hline $12 / 01$ & $10-20$ & 4,60 & 0,40 & 6,60 & 0,09 \\
\hline \multicolumn{6}{|c|}{ Propriedade C } \\
\hline $12 / 00$ & $00-05$ & 5,40 & 0,00 & 11,27 & 0,31 \\
\hline $12 / 01$ & $00-05$ & 5,60 & 0,00 & 13,10 & 0,36 \\
\hline 1260 & $05-10$ & 5,20 & 0,00 & 7,40 & 0,21 \\
\hline $12 / 01$ & $05-10$ & 5,50 & 0,00 & 11,00 & 0,18 \\
\hline 1200 & $10-20$ & 5,10 & 0,00 & 6,06 & 0,11 \\
\hline $12 / 01$ & $10-20$ & 5,40 & 0,00 & 9,60 & 0,16 \\
\hline \multicolumn{6}{|c|}{ Proprie dade D } \\
\hline $12 / 00$ & $00-05$ & 5,10 & 0,00 & 7,90 & 0,10 \\
\hline $12 / 01$ & $00-05$ & 5,40 & 0,00 & 11,10 & 0,20 \\
\hline $12 / 00$ & $05-10$ & 5,10 & 0,00 & 6,70 & 0,08 \\
\hline $12 / 01$ & $05-10$ & 5,30 & 0,00 & 8,40 & 0,15 \\
\hline $12 / 00$ & $10-20$ & 4,90 & 0,00 & 5,00 & 0,06 \\
\hline $12 / 01$ & $10-20$ & 5,20 & 0,00 & 6,00 & 0,10 \\
\hline \multicolumn{6}{|c|}{ Proprie dade E } \\
\hline 1200 & $00-05$ & 5,20 & 0,00 & 10,50 & 0,35 \\
\hline $12 / 01$ & $00-05$ & 5,60 & 0,00 & 10,90 & 0,37 \\
\hline $12 / 00$ & $05-10$ & 5,00 & 0,00 & 7,80 & 0,27 \\
\hline 1201 & $05-10$ & 5,20 & 0,00 & 4,80 & 0,24 \\
\hline $12 / 00$ & $10-20$ & 4,80 & 0,30 & 5,90 & 0,20 \\
\hline $12 / 01$ & $10-20$ & 4,80 & 0,30 & 4,50 & 0,18 \\
\hline \multicolumn{6}{|c|}{ Proprie dade F } \\
\hline 12,00 & $00-05$ & 4,90 & 0,00 & 5,30 & 0,19 \\
\hline $12 / 01$ & $00-05$ & 5,60 & 0,00 & 13,40 & 0,24 \\
\hline $12 / 00$ & $05-10$ & 5,50 & 0,00 & 11,00 & 0,28 \\
\hline 1201 & $05-10$ & 5,60 & 0,00 & 12,50 & 0,18 \\
\hline $12 / 00$ & $10-20$ & 4,60 & 0,50 & 9,20 & 0,16 \\
\hline 1201 & $10-20$ & 5,60 & 0,00 & 11,40 & 0,17 \\
\hline
\end{tabular}

$\mathrm{O} \mathrm{pH}$ dos solos aumentou na segunda avaliação em todas as áreas estudadas e em todas as profundidades, demonstrando que apesar da aplicação de calcário ser realizada superficialmente em sistema de plantio direto, a reação no solo ocorreu de maneira uniforme, possivelmente devido à rotação de cultu- ras. Mesmo nas propriedades B e E, onde não houve aplicação de calcário durante o período de estudo os teores se elevaram.

Já os teores de $\mathrm{Ca}^{+2}+\mathrm{Mg}^{+2}$, com exceção das propriedades B na camada superficial e E nas camadas mais profundas, aumentaram de um ano para 
o outro. Neste caso, as maiores concentrações apresentaram-se nas camadas mais superficiais devido à aplicação superficial do calcário em sistema de plantio direto. A neutralização do alumínio tóxico também pode ser verificada nas propriedades A, B, E e F.

Na propriedade B observa-se diminuição nos teores de $\mathrm{K}^{+}$, após um ano de monitoramento. A quantidade de $45 \mathrm{~kg} . \mathrm{ha}^{-1}$ de $\mathrm{K}_{2} \mathrm{O}$ adicionada ao sistema durante o período da pesquisa possivelmente não supriu a demanda das culturas de sorgo + milheto e aveia + azevém (Tabela 1). Já na propriedade E a adição de 80 kg.ha ${ }^{-1}$ de $\mathrm{K}_{2} \mathrm{O}$ aumentou o teor de $\mathrm{K}$ na superfície, mas os teores nas camadas mais profundas diminuíram. Nas demais propriedades, os teores aumentaram após um ano de monitoramento, até mesmo na propriedade $\mathrm{D}$ onde a aplicação de apenas 38 $\mathrm{kg} . \mathrm{ha}^{-1}$ de $\mathrm{K}_{2} \mathrm{O}$ e o cultivo da soja poderiam ter reduzido os teores disponíveis, considerando que a cultura da soja extrai cerca de $20 \mathrm{~kg} \cdot \mathrm{ha}^{-1}$ de $\mathrm{K}_{2} \mathrm{O}$ para cada tonelada de grãos produzida (EMBRAPA/CNPSo, 2001).

Os teores de fósforo apresentaram variações entre diferentes épocas e profundidades dentro de cada propriedade estudada (Tabela 6).

\section{TABELA 6 - Resultado de análise de P, C e V (\%) em duas épocas e três profundidades de solos no sistema de integração lavoura-pecuária em seis propriedades rurais da região de Guarapuava/PR. 2001.}

Table 6 - Result of analysis of $\mathrm{P}, \mathrm{C}$ and $\mathrm{V}(\%)$ at two times and three depths of soil in crop-cattle raising integration system in six properties of the Guarapuava/PR region. 2001.

\begin{tabular}{|c|c|c|c|c|}
\hline $\begin{array}{c}\text { Epoca } \\
\text { (mêsiano) }\end{array}$ & $\begin{array}{l}\text { Profundidade } \\
\text { (cm) }\end{array}$ & $\begin{array}{c}\mathrm{P} \\
\left(\mathrm{mg} \cdot \mathrm{dm} \mathrm{m}^{-3}\right.\end{array}$ & $\begin{array}{c}\mathrm{C} \\
\left(\mathrm{g} \cdot \mathrm{d} \mathrm{m}^{-3}\right)\end{array}$ & $\begin{array}{l}\mathrm{V} \\
(\%)\end{array}$ \\
\hline \multicolumn{5}{|c|}{ Proprie dade A } \\
\hline 1200 & $00-05$ & 3,7 & 32,3 & 53,73 \\
\hline 1201 & $00-05$ & 4,9 & 34,7 & 64,57 \\
\hline 1200 & $05-10$ & 4,4 & 29,3 & 38,23 \\
\hline 1201 & $05-10$ & 6,0 & 32,3 & 53,06 \\
\hline 1200 & $10-20$ & 1,5 & 23,2 & 27,93 \\
\hline 1201 & $10-20$ & 2,1 & 28,7 & 36,12 \\
\hline \multicolumn{5}{|c|}{ Fropriedade B } \\
\hline 12,00 & $00-05$ & 7,6 & 42,0 & 57,06 \\
\hline 1201 & $00-05$ & 6,8 & 38,3 & 65,11 \\
\hline 1200 & $05-10$ & 3,5 & 34,7 & 57,21 \\
\hline 12,01 & $05-10$ & 8,8 & 30,5 & 65,42 \\
\hline 1200 & $10-20$ & 2,5 & 31,1 & 35,13 \\
\hline 1201 & $10-20$ & 3,5 & 26,3 & 42,64 \\
\hline \multicolumn{5}{|c|}{ Propriedade C } \\
\hline 1200 & $00-05$ & 5,4 & 33,5 & 65,16 \\
\hline 12,01 & $00-05$ & 6,2 & 44,4 & 74,53 \\
\hline 1200 & $05-10$ & 5,5 & 31,1 & 51,94 \\
\hline 1201 & $05-10$ & 4,9 & 40,8 & 67,43 \\
\hline 1200 & $10-20$ & 2,3 & 30,0 & 44,36 \\
\hline 1201 & $10-20$ & 2,8 & 38,9 & 61,15 \\
\hline \multicolumn{5}{|c|}{ Proprie dade D } \\
\hline 1200 & $00-05$ & 5,7 & 33,5 & 52,63 \\
\hline 12,01 & $00-05$ & 6,5 & 38,3 & 69,33 \\
\hline 1200 & $05-10$ & 5,2 & 32,3 & 50,30 \\
\hline 1201 & $05-10$ & 5,4 & 35,9 & 59,58 \\
\hline 1200 & $10-20$ & 3,0 & 25,7 & 41,27 \\
\hline 1201 & $10-20$ & 4,6 & 29,3 & 47,66 \\
\hline \multicolumn{5}{|c|}{ Proprie dade E } \\
\hline 1200 & $00-05$ & 9,8 & 37,7 & 61,76 \\
\hline 1201 & $00-05$ & 14,1 & 41,4 & 69,27 \\
\hline 12,00 & $05-10$ & 6,2 & 34,7 & 52,85 \\
\hline 1201 & $05-10$ & 6,2 & 36,5 & 54,84 \\
\hline 1200 & $10-20$ & 2,8 & 37,7 & 38,61 \\
\hline 12,01 & $10-20$ & 3,9 & 31,1 & 37,50 \\
\hline \multicolumn{5}{|c|}{ Pro prie dade F } \\
\hline 1200 & $00-05$ & 3,7 & 26,3 & 43,26 \\
\hline 1201 & $00-05$ & 20,7 & 41,4 & 74,78 \\
\hline 12,00 & $05-10$ & 10,4 & 37,1 & 64,53 \\
\hline 1201 & $05-10$ & 9,1 & 37,1 & 71,72 \\
\hline 1200 & $10-20$ & 10,2 & 28,7 & 50,98 \\
\hline 1201 & $10-20$ & 10,4 & 34,7 & 69,82 \\
\hline
\end{tabular}


Na propriedade F, a adição de 112 kg.ha- ${ }^{-1}$ de $\mathrm{P}_{2} \mathrm{O}_{5}$ elevou o teor de 3,7 para 20,7 $\mathrm{mg} \cdot \mathrm{dm}^{-3}$ no segundo ano de monitoramento na camada superficial. Entretanto, na camada de 5 a $10 \mathrm{~cm}$ de profundidade o teor que era de $10,4 \mathrm{mg} \cdot \mathrm{dm}^{-3}$ no primeiro ano, passou para 9,1 $\mathrm{mg} \cdot \mathrm{dm}^{-3}$ no segundo ano.

A única propriedade que apresentou redução do teor de carbono após um ano de monitoramento foi a B. Nesta propriedade foi adicionado ao sistema $253 \mathrm{~kg}$ de N.ha-1 . A quantidade de $\mathrm{N}$ adicionada possivelmente alterou a relação $\mathrm{C}: \mathrm{N}$, podendo ter alterado a velocidade de decomposição da matéria orgânica presente (TROECH; THOMPSON, 1993). É certo que muitos outros fatores podem ter influenciado neste caso, como, por exemplo, a pressão de pastejo que não foi estudada neste experimento. O processo de degradação de matéria orgânica pode ser promovido pelo manejo inadequado de biomassa que implica no aumento das taxas de perdas de nutrientes (ASSMANN et al., 2002).

Russel (1996) apud Assmann et al. (2002) afirma que o incremento inicial no teor de matéria orgânica ocorre na superfície do solo, mas, com o tempo, existe um deslocamento no perfil, podendo ser observado até $20 \mathrm{~cm}$ de profundidade. Na propriedade C observa-se este aumento em profundidade e, de maneira geral, todos os solos são muito ricos em matéria orgânica, o que não os caracteriza como solos orgânicos já que são de textura argilosa (Tabela 1).

A saturação por bases (V\%) aumentou em todas as áreas, sendo que os solos das seis propriedades estudadas podem ser classificados como eutróficos quando considerada a segunda avaliação na camada de 0 a $10 \mathrm{~cm}$ (TOMÉ JÚNIOR, 1997). Sendo assim, as quantidades médias de nutrientes adicionadas ao sistema durante o período de realização deste estudo (210 kg de N.ha-1, $113 \mathrm{~kg}$ de $\mathrm{P}_{2} \mathrm{O}_{5} \cdot \mathrm{ha}^{-1}$ e $95 \mathrm{~kg}$ de $\left.\mathrm{K}_{2} \mathrm{O} \cdot \mathrm{ha}^{-1}\right)$, as rotações de culturas e o sistema de pastejo (média de 4 cabeças.ha ${ }^{-1}$ ) utilizados nas propriedades estudadas (Tabelas 1 e 2) mostram-se adequados para a manutenção e o incremento da fertilidade dos solos estudados.

Entretanto, o impacto ambiental do sistema e o retorno econômico devem ser associados à fertilidade do solo em pesquisas futuras.

\section{Considerações Finais}

O nível de fertilidade dos solos aumentou após um ano de manejo em sistema de integração lavoura-pecuária, quando são adotados procedimentos técnicos adequados.

\section{Agradecimento}

À empresa Monsanto, pelo auxílio financeiro.

\section{Referências}

ASSMANN, T. S.; CASSOL, L. C.; ASSMANN, A. L. Manejo da fertilidade do solo (ciclagem de nutrientes) em sistemas de integração lavoura-pecuária. In: ENCONTRO DE INTEGRAÇÃO LAVOURA-PECUÁRIA NO SUL DO BRASIL, 1., Pato Branco, 2002. Anais... Pato Branco: CEFET-PR, 2002. p. 61-84.

BRUGINSKI, D. H. Nitrogênio em cobertura no cultivo de girassol em sistema de plantio direto na palha. Curitiba: 1999. 62f. Dissertação (Mestrado em Agronomia) - Universidade Federal do Paraná.

EMBRAPA/CNPSO. Tecnologia de produção de soja - Paraná - 2001/2002. Londrina: Embrapa Soja, 2001. 281p. (Documentos/Embrapa Soja, n.166).

LOTERO, J.; WOODHOUSE J. R., W. W.; PETERSEN, R. G. Local effect on fertility of urine voided by grazing cattle. Agronomy Journal, Madison, p. 262-265, 1966.

MORAES, A., et al. Sistemas de produção: integração lavoura-pecuária no $3^{\circ}$ planalto do Paraná. In: REUNIÃO DO GRUPO TÉCNICO DE FORRAGEIRAS DO CONE SUL - ZONA CAMPOS: DINÂMICA DA VEGETAÇÃO EM ECOSSISTEMAS PASTORIS, 18., Guarapuava, 2000. Anais... Guarapuava: CPAF/ FAPA, 2000. p. 6-22.

MORAES, A., et al. Integração lavoura-pecuária no Sul do Brasil. In: ENCONTRO DE INTEGRAÇÃO LAVOURA-PECUÁRIA NO SUL DO BRASIL, 1., Pato Branco, 2002. Anais... Pato Branco: CEFET-PR, 2002. p. 3-42.

NABINGER, C.; MARASCHIN, G. E.; MORAES, A. Pasture related problems in beef cattle production 
in Southern Brazil. In: SIMPÓSIO INTERNACIONAL "GRASSLAND ECOPHYSIOLOGY AND GRAZING ECOLOGY”, Curitiba, 1999. Anais... Curitiba: UFPR. 1999. p. 23-48.

RHEINHEIMER, D. S.; ANGHINONI, I. Distribuição do fósforo inorgânico em sistemas de manejo de solo. Pesquisa Agropecuária Brasileira, v. 36, n. 1, p. 151-160, 2001.

SANTOS, H. P.; FONTANELI, R. S.; TOMM, G. O. Efeito de sistemas de produção de grãos e de pastagens sob plantio direto sobre o nível de fertilidade do solo após cinco anos. Revista Brasileira de Ciência do Solo, Campinas, v. 25, p. 645653, 2001.
TOMÉ JÚNIOR, J. B. Manual para interpretação de análise de solo. Guaíba: Agropecuária, 1997. $247 \mathrm{p}$.

TROECH, F. R.; THOMPSON, L. M. Soils and soil fertility. 5. ed. Oxford: Oxford University Press, 1993. $462 \mathrm{p}$.

VALPASSOS, M. A. R. et al. Efeitos de sistemas de uso e manejo na atividade microbiana, densidade e propriedades químicas do solo. Pesquisa Agropecuária Brasileira, v. 36, n. 12, p.15391545, 2001.

WONG, M. T. F.; NORTCLIFF, S.; SWIFT, R. S. Methods for determination the acid ameliorating capacity of plant residue compost, urban waste compost, farmyard manure, and peat applied to tropical soils. Commun. Soil Pl. Anal. v. 29, p. 2927-2937, 1998. 